\title{
Molecular allergy diagnostics
}

using IgE singleplex

determinations: methodological and practical considerations for use in clinical routine

\section{Part 18 of the Series Molecular Allergology}

\author{
Jörg Kleine-TebBe', ThiLo JaKob² \\ 'Allergy \& Asthma Center Westend, Outpatient Clinic \& Research Center Hanf, Ackermann-Simon, and Kleine- \\ Tebbe, Berlin, Germany; ${ }^{2}$ Allergy Department and Allergy Research Group, Department of Dermatology and \\ Venereology, Freiburg University Hospital, Freiburg, Germany
}

\begin{abstract}
Allergen molecules (synonyms: single allergens, allergen components) open up new horizons for the targeted allergen-specific diagnostics of immunoglobulin $\mathrm{E}$ (IgE) in singleplex determination. The following rationales support the targeted use of allergen molecules and, more importantly, improve test properties: (1) increased test sensitivity ("analytical sensitivity"), particularly when important allergens are under-represented or lacking in the extract; (2) improved test selectivity (analytical specificity), particularly when the selected IgE repertoire against an allergen yields additional information on: (a) potential risk, (b) possible cross-reactivity, or (c) primary (species-specific) sensitization. However, the appropriate indication for the use of single allergens can only be established on a case-by-case basis (depending on the clinical context and previous history) and in an allergen-specific manner (depending on the allergen source and the single allergens available), rather than in a standardized way.

Numerous investigations on suspected food allergy, insect venom allergy, or sensitization to respiratory allergens have meanwhile demonstrated the
\end{abstract}

successful use of defined molecules for allergen-specific singleplex IgE diagnosis. Specific IgE to single allergens is limited in its suitability to predict the clinical relevance of sensitivity on an individual basis. In food allergies, one can at best identify the relative risk of a clinical reaction on the basis of an IgE profile, but no absolutely reliable prediction on (future) tolerance can be made. Ultimately, the clinical relevance of all IgE findings depends on the presence of corresponding symptoms and can only be assessed on an individual basis (previous history, symptom log, and provocation testing with the relevant allergen source where appropriate). Thus, also in molecular allergology, the treating physician and not the test result should determine the clinical relevance of diagnostic findings.

Cite this as Kleine-Tebbe J, Jakob T. Molecular allergy diagnostics using IgE singleplex determinations: methodological and practical consideration for use in clinical routine - Part 18 of the Series Molecular Allergology. Allergo J Int 2015;24:185-97 DOI: 10.1007/s40629-015-0067-z

\section{Key words} IgE - Allergen Molecular allergy diagnostics Componentbased diagnostics In-vitro test

\section{Submitted}

May 4, 2015

\section{Accepted}

May 26, 2015

German version www.springermedizin.de/ allergo-journal 


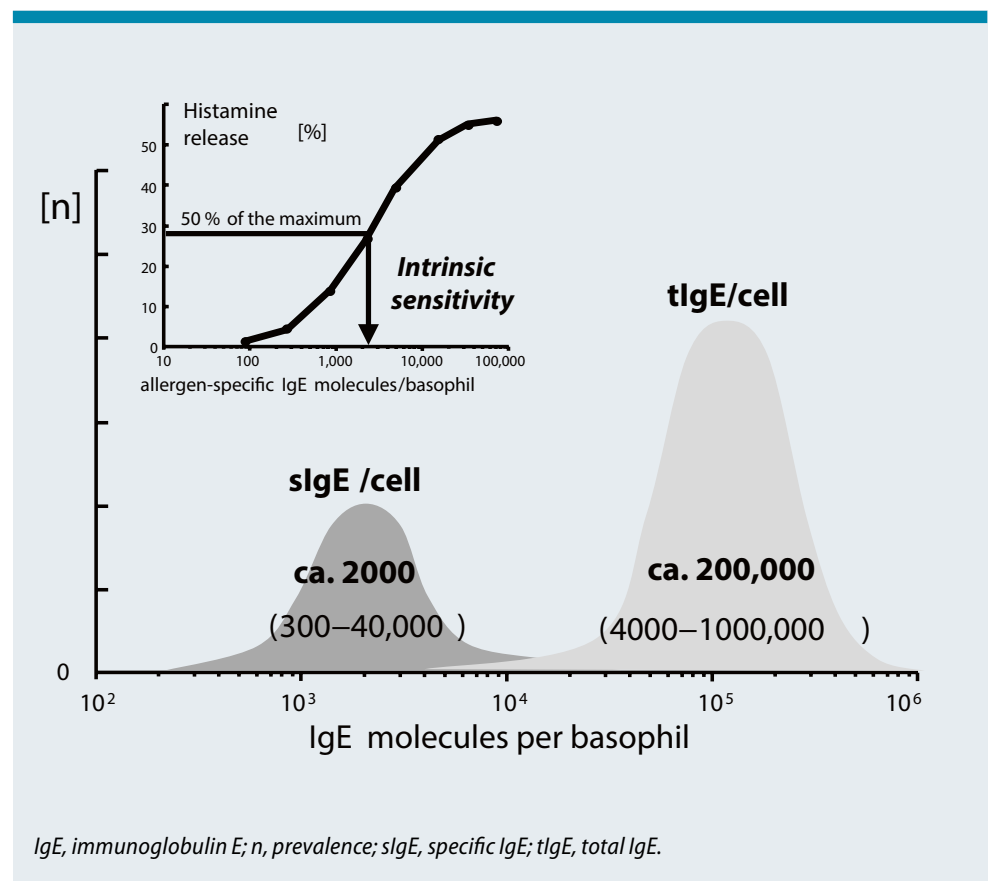

Fig. 1: FceRI-bound IgE on effector cells. Light gray area: total bound IgE/cell (number of FcERI occupied by IgE with population-based distribution) on basophilic leukocytes. Dark gray area: specific IgE/cell required for halfmaximal cell activation (intrinsic sensitivity of basophils with populationbased distribution). The distribution of both variables is approximately normal and can differ significantly; evidently, a fraction (ca. 1\%) of bound total lgE is sufficient for half-maximal allergen specific activation. For this reason, the ratio of specific to total $\lg \mathrm{E}$ is interesting in terms of interpretation. Inset top left: individual mediator release as a function of cell-bound specific $\lg \mathrm{E}$; basis for the population-based normal distributions illustrated in the lower part of the figure. mary). Approximately half of IgE is found as free IgE in the vascular bed, while the other half is bound by the IgE receptors of a variety of cells. The high-affinity IgE receptor (FceRI) on tissue-bound mast cells and basophils is the most important binding partner (approximately 100,000-250,000 FceRI/ basophilic leukocyte; Fig. 1) in immediate-type allergic reactions. Although free serum IgE has a halflife of only a few days, FceRI-bound IgE persists for approximately 2 months due to a slow off-rate. Thus, it is not free but rather cell-bound IgE that is essential for the effector phase of the allergic reaction. Upon re-exposure to allergens specific IgE antibodies are cross-linked, either in pairs or as large aggregates. It takes on average 2000 cross-linked IgE molecules to induce a half-maximal cell response (e.g., histamine release, Fig. 1), i.e., only a fraction of total cell-bound IgE (200,000 molecules/basophil, range 100,000-250,000). For this reason, basophil tests have an extremely high test sensitivity. The activation status of effector cells can be quantified by the expression of specific surface markers (CD63, CD203c) using flow cytometry; basophilic leukocytes from fresh blood are generally used here, since they are easier to isolate (basophil activation test, BAT).

The following variables have a significant effect on the dose-dependent activation of basophilic leukocytes [4]:

- The total amount of cell-bound IgE

- The ratio of specific IgE to total IgE (as little as

$1 \%$ is sufficient for half-maximal activation of effector cells, see above)

- The number of epitope-specific antibodies capable of binding (clonality)

— The binding strength between individual IgE antibodies and the allergen (affinity)

_ The total number of multivalent specific IgE binding sites that bind strongly to the allergen (avidity)

- The ratio of low- to high-affinity IgE antibodies IgE stabilizes the continuously newly synthesized FceRI receptors at the cell surface [5]. In this way, the level of total IgE passively regulates the number of its receptors and thus also the amount of cell-bound IgE [6]. The complex variables involved [7], besides the allergen-specific IgE level, explain why various sensitization tests (specific IgE, titrated skin test, dose-dependent BAT) correspond well qualitatively (correspondence between positive or negative results) but not quantitatively $[8]$.

\section{$\lg \mathrm{E}$, IgE receptors, and the effector phase of allergic reactions: background information and relevance in IgE diagnostics}

As the least abundant human antibody, IgE was not discovered until 1966 (see [3] for an historical sum-

\section{The IgE repertoire: a phenomenon with complex variables}

The IgE synthesized by plasma cells is directed against (glyco)protein surface structures. The 


$\begin{array}{ll}\text { Abbreviations } \\ \text { BAT } \quad \text { Basophil activation test } \\ \text { CAST } & \text { Cellular allergen stimulation test } \\ \text { CCD } & \text { Cross-reactive carbohydrate determinants } \\ \text { CRD } & \text { Component resolved diagnostics } \\ \text { IgE } & \text { Immunoglobulin E } \\ \text { LoB } & \text { Limit of blank } \\ \text { LoD } & \text { Limit of detection } \\ \text { LoQ } & \text { Limit of quantitation } \\ \text { NPV } & \text { Negative predictive value } \\ \text { PPV } & \text { Positive predictive value } \\ \text { RAST } & \text { Radioallergosorbent test } \\ \text { RiliBÄK Laboratory Guidelines of the German } \\ \text { Redical Association (Bundesärztekammer) } \\ \text { ROC } & \begin{array}{l}\text { curve Receiver operating characteristic } \\ \text { curve }\end{array} \\ \text { SIgE } & \text { Specific immunoglobulin E } \\ \text { WDEIA } & \text { Wheat-dependent exercise-induced } \\ \text { anaphylaxis } & \text { World Health Organization } \\ & \end{array}$

more alike and abundant the common binding sites (epitopes), the likelier it is that specific $\operatorname{IgE}$ will bind to allergens of similar structure-the basis of serological or clinical cross-reactivity.

Polyclonal IgE antibodies differ in terms of their binding strength (avidity/affinity) and recognition of specific epitopes [9]. The resulting IgE repertoire, e.g., against one allergen molecule, is therefore made up of a multitude of antibodies with differing epitope specificity and binding strength. In the course of the immune response to an allergen, the repertoire can expand and the binding strength increase through the recognition of further epitopes. To date, it has only been possible to investigate the variables described (epitope specificity, avidity, polyclonality) under experimental conditions, not in routine tests. Thus, even modern quantitative singleplex tests for specific IgE determination using individual allergen molecules can recognize only the total quantity of the polyclonal IgE response ("the scale of the iceberg") in the best case, while additional parameters of the allergen-specific repertoire ("the number and height of the various tips of the iceberg") continue to remain hidden to routine diagnostics [10].

\section{Techniques to detect sensitization in routine} diagnostics

In routine diagnostics, sensitization tests serve to detect IgE either:

- directly or

-indirectly.

The following methods are available to detect sensitization in IgE-mediated reactions and disease:

1. Skin prick tests (in selected cases, intradermal test; [11])

2. Serum IgE determination (allergen-specific IgE, total IgE; [12])

3. Basophil activation tests (BAT, cellular antigen stimulation test, CAST) only in selected indications [13]

While serum IgE determination directly measures free IgE, the skin prick test and BAT yield indirect information on mast cell- and basophil-bound IgE. As such, they are comparable in terms of the diagnostic (qualitative) information they yield, even though there can be significant quantitative variation between results, particularly in the case of different allergen sources and due to the variables mentioned above [8].

\section{Technological basis of IgE determination}

Solid phase immunoassays for the routine diagnosis of specific IgE have been available since the early 1970s. Initially, radio-immunological methods (radioallergosorbent test, RAST) consisted of immobilized allergen extracts to activated paper disks in order to bind specific IgE from the sera of allergy sufferers. Today, assay signals are no longer detected by means of a radioactive label, but rather by means of enzyme labeling or fluorometry; moreover, molecules are also increasingly used in this context.

\section{Test design and test components}

Modern immunoassays to determine allergen-specific IgE antibodies (overview in Tab. 1) comprise the following components [14]:

a. Reaction vessel: Plastic (polyethylene) or glass test tubes, plastic microtiter plate with wells, plastic stick or bead, polyethylene cap with sponge-like matrix

b. Allergen-containing reagent: solid phase allergosorbent to a or liquid-phase labeled allergen

c. Anti-IgE-Fc antibody (detection antibody specific to the constant Fc fragment of IgE)

d. Calibration system: e.g., reference serum with defined IgE amount in order to generate a total IgE calibration curve

e. Reaction buffer: salt- and protein-containing solutions for constant $\mathrm{pH}$ values and constant protein matrix to ensure minimum nonspecific binding 
f. Human serum with specific IgE antibodies and negative serum controls

g. Data processing system (software or algorithm) The allergen-containing reagent $(b)$ is considered the most complex component of the test variables, irrespective of extracts of biological origin or single defined allergen molecules are involved.

The second and equally important component is the anti-human IgE reagent (c).

The calibration system (d) is the third key component of IgE determination.

Since there are no internationally accepted calibration standards for allergen-specific IgE tests, a total IgE calibration curve is used to convert the units measured to be expressed as quantitative allergen-specific IgE antibody levels (Fig. 2): $\mathrm{kU}_{\mathrm{A}} / \mathrm{l}$ (where "A" stands for "allergen-specific", thereby distinguishing units from the internationally standardized $\mathrm{kU} / \mathrm{l}=\mathrm{IU} / \mathrm{ml}$ for total IgE determination). To this end, a reference curve calibrated to the official WHO standard for total IgE (formerly WHO $75 / 502$, currently WHO 11/234) is generated following each assay run according to the specifications of the manufacturers of these diagnostic products. The measurement signals thus obtained for allergen-specific $\operatorname{IgE}$ are then converted into the corresponding units $\left(\mathrm{kU}_{\mathrm{A}} / \mathrm{l}\right)$ with the help of this total IgE reference curve ("heterologous" cal-

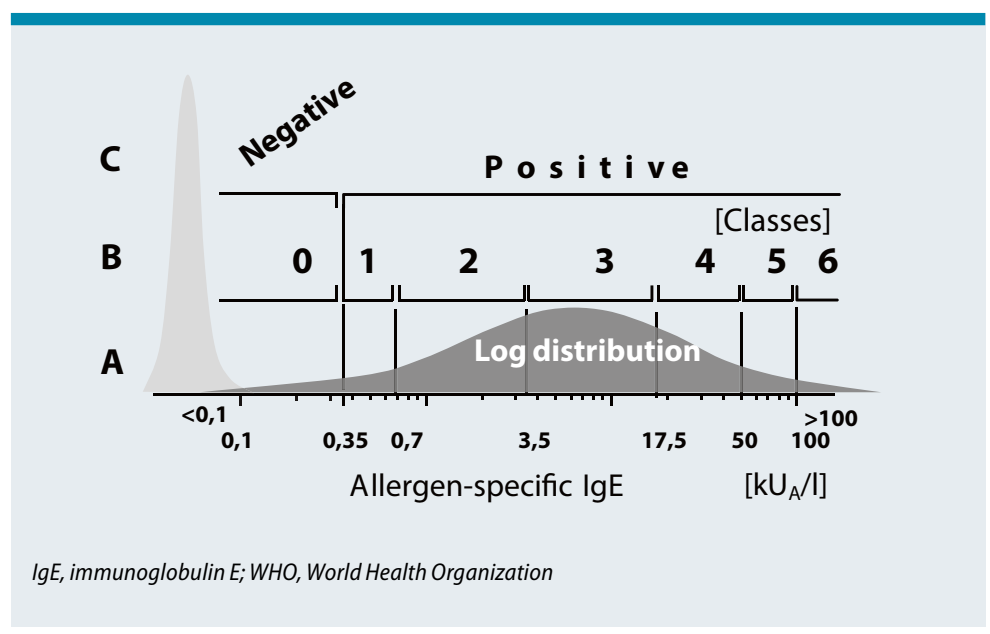

Fig. 2: Options for the evaluation of logarithmically distributed allergen-specific IgE levels. A quantitative; $B$ semi-quantitative (since entry into force of the German Medical Association guideline, Richtlinie der Bundesärztekammer (RiliBÄK), this term is no longer provided for; specific lgE levels given only in classes are considered as qualitative evaluations); $C$ qualitative. Allergen-specific IgE levels expressed as units of specific $\lg \mathrm{E}, \mathrm{kU}_{\mathrm{A}} / \mathrm{l}$ (A stands for allergen-specific), using WHO standards for total IgE determination (heterologous calibration). Light gray area: population of serum samples with no allergen-specific lgE (levels fall below the detection limit of $0.1 \mathrm{kU}_{\mathrm{A}} / \mathrm{l}$ ). Dark gray area: population of positive serum samples with logarithmic (hypothetically normal) distribution of allergen-specific lgE levels above the detection limit of $0.1 \mathrm{kU}_{\mathrm{A}} / \mathrm{l}$ ibration). As part of this process, one assumes a comparable binding strength between the allergen (extract) and specific IgE or between the primary anti-IgE antibodies used for the reference curve and total IgE-a source of error to a certain extent, which nevertheless needs to be accepted for heterologous calibration and which can cause deviations of maximum $10 \%$. The randomly assigned "classes" that have evolved over time serve to semi-quantitatively and broadly categorize IgE concentrations and, in the authors' view, play only a minor role today. The test systems currently available, as well as their test principles, are listed in Tab. 1.

\section{Detection thresholds in slgE determination}

The lower detection threshold for specific IgE was formerly $0.35 \mathrm{kU}_{\mathrm{A}} / \mathrm{l}$. The sensitivity of IgE test methods is now higher thanks to more sensitive calibration and improved resolution of low IgE values. Thus, modern specific $\operatorname{IgE}$ immunoassays now yield values below 0.35 down to $0.1 \mathrm{kU}_{\mathrm{A}} / \mathrm{l}$ (Fig. 2). This range is particularly informative and relevant when total IgE is extremely low $(<20,<10,<5 \mathrm{kU} / \mathrm{l})$. The upper detection limit is $100 \mathrm{kU}_{\mathrm{A}} / \mathrm{l}$ for most specific-IgE detection methods. Therefore, sera with higher specific IgE should be measured in diluted form (1:10) in order to determine the actual value after multiplying $\times 10$.

\section{Specific IgE/total lgE ratio}

Certain modern assays have shown that the unit for total IgE $(\mathrm{kU} / \mathrm{l})$ corresponds to the heterologously calibrated units for allergen-specific $\operatorname{IgE}\left(\mathrm{kU}_{\mathrm{A}} / \mathrm{l}\right)$ [15]. Working on this assumption, both variables, specific and total IgE, can be directly compared and used to improve interpretation [16]. The ratio of specific IgE to total IgE (also referred to as antibody-specific activity [16]) is particularly important in the case of: _Extremely low total IgE levels (e.g., $<20 \mathrm{kU} / 1,<10$ $\mathrm{kU} / \mathrm{l},<5 \mathrm{kU} / \mathrm{l})$ :

-For instance, in some atopic patients with unusually low total IgE

- Non-atopic patients with IgE sensitization to particular allergens, e.g., insect venom or occupational allergens

_Extremely high total IgE levels:

_For instance, in atopic patients with currently or previously manifest atopic dermatitis

_In patients with other causes of extremely high total IgE [12]

It is important to bear in mind that normal distribution of $\operatorname{IgE}$ is not linear, but rather logarithmic and needs to be represented using logarithmic scales (Fig. 2).

The ratio of specific IgE to total IgE in serum is found in the same way on effector cells (mast cells, basophils). If specific IgE is given relative (e.g., in 


\section{Tab 1: Different test principles for determining specific IgE antibodies using singleplex assays}

\begin{abstract}
IgE assay format
Short description

Solid-phase assay

Solid-phase assays have been established for IgE determination for many years. The allergens coupled to a solid substrate directly bind all allergen-specific antibodies (lgE, $\mathrm{IgG}$, etc.); a washing procedure removes unbound antibodies. Bound specific lgE antibodies are then determined using radiolabeled anti-IgE antibodies. The latter, together with marker labels (fluorescence, chemiluminescence), enable the quantitation of bound specific lgE
\end{abstract}

\section{Liquid-phase}

assay

\begin{abstract}
This test format involves the use of liquid and labeled allergens to bind allergen-specific lgE. Following the appropriate washing procedure, the allergen-IgE marker complexes are bound by immobilized reagents (e.g., the biotin-streptavidin system). The use of appropriate substrates likewise enables quantitation of primarily bound specific $\lg E$ on the allergens used
\end{abstract}

Reverse IgE assay

With this test system, all $\lg E$ antibodies (e.g., contained in serum) are bound by immobilized anti-lgE antibodies in a first step. Following the removal of unbound antibodies (e.g., IgG), allergen-specific IgE can be identified by adding appropriately labeled liquid allergens. By labeling the allergens, it is possible to quantify specific bound antibodies

\section{Advantages}

Disadvantages

In the case of a large surface area of the solid phase used and surplus allergens/allergen sources, complete binding of all specific $\lg \mathrm{E}$ antibodies is possible (prerequisite of true quantitation). However, low-affinity lgE antibodies are also bound

Rapid binding kinetics due to the liquid phase
In the case of low surface area of the solid phase (e.g., paper disk) and no surplus allergens/allergen sources, true quantitation of specific $\lg E$ is not possible and competitive inhibition of the lgE signal by allergen-specific IgG antibodies occurs (particularly in high titers, e.g., after allergen-specific immunotherapy)

True quantitation of specific $\lg E$ not possible in the absence of surplus allergens/allergen sources

No inhibition caused by the high proportion of allergen-specific IgG antibodies imited binding capacity particularly in the case of extremely high total $\lg \mathrm{E}(>2000 \mathrm{kU} / \mathrm{l})$

IgE, immunoglobulin E; IgG immunoglobulin G

percent) to total IgE (see Fig. 3 for a more detailed explanation) [16], the values relating to individual total IgE levels are normalized: by taking this step, one can expect better concordance between the relative specific IgE proportion (in percent) and the quantitative analysis of other sensitization tests (skin prick test, BAT).

\section{Isoforms: natural variants of allergen molecules}

Points of criticism on use of allergen molecules relate to their origin or production:

When derived from natural sources, even defined allergens are variable mixtures with multiple molecule variants (isoforms), which bind IgE with varying strength depending on the individual IgE repertoire. Mixing isoforms potentially has the advantage of covering all IgE specificities; however, complex mixtures of this kind are challenging to purify and standardize. Therefore, allergens molecules are predominantly used in recombinant form for molecular IgE diagnostics.

\section{Possible applications for allergen molecules in IgE diagnostics}

Serological in vitro diagnosis can be modified in different of ways using single allergens: a. Allergen molecules are used individually as reagents for specific IgE determination (currently the most frequent application).

b. Selected allergens are combined as reagents for specific IgE determination (combination of im-

\section{Definition of terms used to measure test method efficacy}

Analytical sensitivity is defined as the slope of an (immuno)assay's calibration curve. The actual sensitivity (lower detection limit) of a test, on the other hand, is determined and expressed today using the following variables [19]: _ Limit of blank (LoB)

- Limit of detection (LoD)

Limit of quantitation (LoQ)

LoB: $L O B$ is defined as the highest apparent test signal obtained from repeated blank measurements (serum sample without $\lg \mathrm{E}$ ): $\mathrm{LoB}=$ meanblank + 1.645 (SDblank).

LoD: LoD refers to the weakest signal or lowest concentration of specific IgE antibodies reliably determined from the test: $L O D=L o B+1.645$ (SDlow concentration sample).

LoQ: LoQ is the lowest concentration of specific IgE antibodies that can be reliably detected within a predefined precision. LoQ may be equivalent to or higher than LoD. 
portant marker allergens, such as $\mathrm{Ph} 1 \mathrm{p} 1$ and $\mathrm{Ph} 1$ p 5, or cross-allergens such as Phl p 7 and Phl p 12).

c. All available individual components of an allergen source can be used as a mix instead of a complex allergen extract (theoretically possible, but not yet implemented due to complexity, costs, and questionable use).

d.Individual components can be added to allergen extracts ("spiking") to increase test sensitivity (e.g., in the case of under-represented components).

Variant (a) enables the targeted and precise differentiation of sensitizations by means of single allergens. This procedure has also known as component-specific or component-resolved diagnostics (CRD) [17] and currently plays the largest role in molecular allergy diagnostics (typical case studies in [18]). A selection of the single allergens currently available for IgE determination using singleplex assays and their significance in routine diagnostics has been summarized in a separate table (Tab. S1) and is available as an online supplement under http://link.springer.com/journal/40629 .

\section{Distinction between purified and recombinantly produced components}

An important crossroad for the manufacturers of diagnostic products is the decision whether to use purified natural single allergens with all their variants (isoforms) or whether to select a single, recombinantly produced protein. The latter should be representative and have the major IgE binding sites in order to be able cover, as far as possible, all allergy sufferers sensitized to this allergen molecule.
This problem does not apply when natural components are used, since these generally contain all molecule variants occurring in natural allergen sources. It is only important to ensure here that the preparations do not contain any impurities with other allergens. This is particularly challenging if the allergen to be purified is available in very small quantities in the allergen source, while other allergens are present in high concentrations. A typical example would be allergens in bee venom (Api m 3, Api $\mathrm{m}$ 5, Api $\mathrm{m} \mathrm{10)}$, which are present at less than $1 \%$ of the venom dry weight, while Api m 4 (melittin), with more than $40 \%$ of the venom dry weight, render clean purification of the above-mentioned allergens virtually impossible.

Another problem with purified natural allergens is encountered when glycoproteins with $\mathrm{N}$-glycan sugar side chains are involved, which are recognized as cross-reactive carbohydrate determinants (CCD) by CCD-specific IgE, thereby falsifying results.

In contrast to the purification of allergens from natural sources, the recombinant production of allergens by selecting the appropriate expression system enables one to circumvent the problem of CCDs. Thus, expressing allergens in Escherichia coli bacteria permits their production without CCD, while production in yeast cells or certain insect cells makes allergens with normal or modified carbohydrate side chains possible.

\section{Technical laboratory evaluation: test sensitivity and analytical specificity (selectivity)}

Test method efficacy is investigated on an international basis using the variables sensitivity and spec-
Fig. 3: Significance of the total and specific immunoglobulin $\mathrm{E}(\mathrm{IgE})$ ratio. Due to the variability of total $\lg \mathrm{E}$ levels, logarithmically distributed specific lgE (dark gray bars) can also be expressed as a relative quantity of total lgE (light gray bars) [16]. This process "normalizes" specific IgE to total IgE on a percentage basis (hatched bars). Primarily the borderline cases (see numerical examples) with particularly low (normal distribution curve, far left) or extremely high total lgE (normal distribution curve, far right) make it clear that specific IgE can only be correctly interpreted once total $\lg E$ is known. This ratio of specific to total $\lg E$ is also found on the surface of effector cells (mast cells, basophil granulocytes), thereby providing the basis for diagnostic ex vivo (basophil activation test, BAT) and in vivo tests (skin prick test, provocation test)

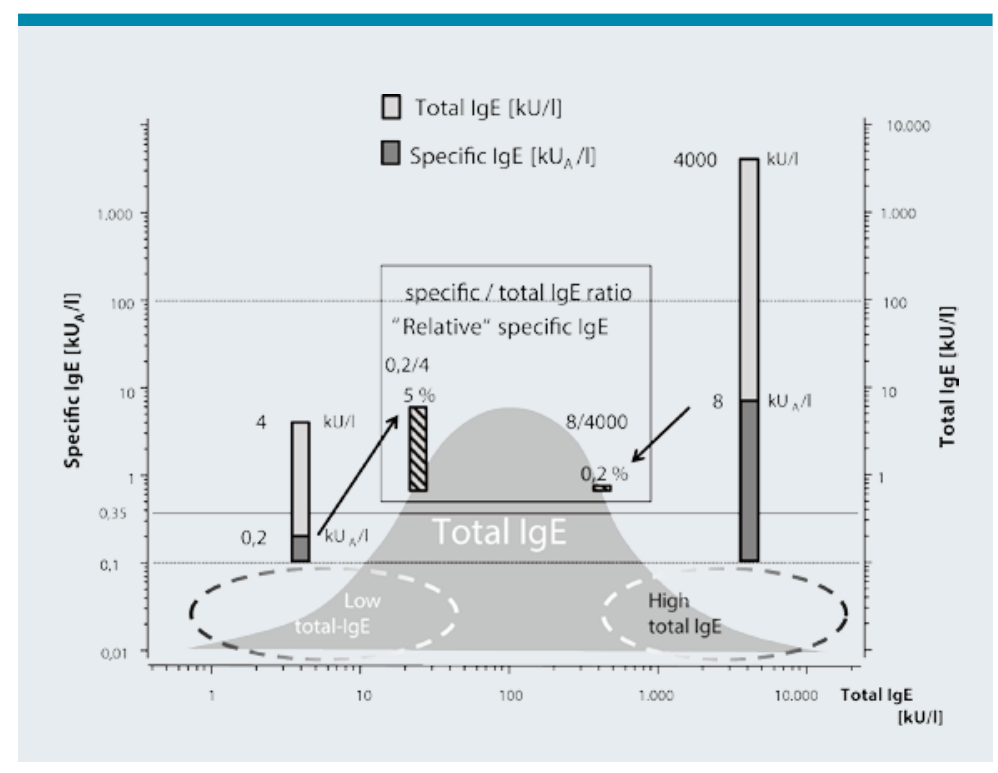


ificity. As part of this process, a distinction is made between two pairs of definitions: analytical and diagnostic sensitivity and specificity.

These definitions are also being introduced in international laboratory guidelines for IgE determination methods and are particularly important when single allergens are used.

Test sensitivity is often improved (i.e., LoQ is lower) when using allergen molecules, particularly if these allergens are under-represented in the natural extract or are entirely absent due to their instability. Greater test sensitivity (lower LoQ) is thus an important argument in support of the use of allergen molecules for the diagnosis of specific IgE (Fig. 4; Tab. 2).

\section{Example}

Sensitization to wheat extract is found in only $20 \%-30 \%$ of patients with wheat-dependent exercise-induced anaphylaxis (WDEIA), while sIgE to Tri a 19 ( $\Omega-5$ gliadin) is detected in $80 \%-90 \%$ of cases. Since the gliadins responsible for WDEIA are not water-soluble, they are not present in sufficient quantities in aqueous wheat extracts. This problem can be avoided by using a recombinantly produced Tri a 19 in the test system.

The analytical specificity of an IgE test method can relate to the specificity of the antibody class on the one hand, whereby the test truly determines IgE and no antibodies of other classes, such as IgA, IgD, IgG or IgM [14].

On the other hand, analytical specificity can relate to a more targeted, more "selective" IgE determination of particular allergen molecules: whereas an allergen extract, as a complex protein mixture, determines the total $\mathrm{IgE}$ repertoire to an allergen source, only a proportion of specific antibodies are determined when using allergen molecules, thereby increasing analytical specificity (selectivity).

This permits more targeted (more analytically specific) detection or exclusion of sensitization particularly in the case of allergen molecules with special characteristics - such as high stability and a relatively high proportion of total protein (e.g., Ara h 2 or Cor a 14) and thus an increased risk for severe reactions to food (peanut or hazelnut).

\section{Example}

More than $10 \%$ of German children and adolescents show specific IgE to peanut extract-caused primarily by pollen-associated cross-reactions. Diagnosis using the stable and risk-related peanut storage protein, Ara h 2, yields elevated values in only a fraction of patients (circa maximum $0.4 \%$ ), thereby providing greater analytical specificity (selectivity) than peanut extract.

\section{Universal arguments for the use of molecular allergens in IgE diagnostics}

Four arguments generally provide plausible support for the use of single allergens (Fig. 4). In this context, particularly the improved test sensitivity (LoQ) and the increased analytical specificity mentioned above help to justify the use of allergen molecules (Fig. 4 and examples in Tab. 2):

1. Provided that allergen molecules (e.g., when present in insufficient proportions or absent in the extract) increase the test sensitivity (LoQ) of $\mathrm{IgE}$ determination, their use is both useful and important.

2. Provided that allergen molecules permit improved analytical specificity (selectivity) by binding a partial amount of the specific IgE repertoire, as well as being linked to additional clinical findings (e.g., increased burden of risk, degree of clinical severity, other associated clinical characteristics), their use is, once again, useful and recommended from a diagnostic perspective.

3. Certain allergen molecules, by binding cross-reactive IgE antibodies, serve as an indicator for cross-sensitizations. In the case of positive results, they indirectly illustrate the lack of analytical specificity of IgE tests against allergen extracts (in

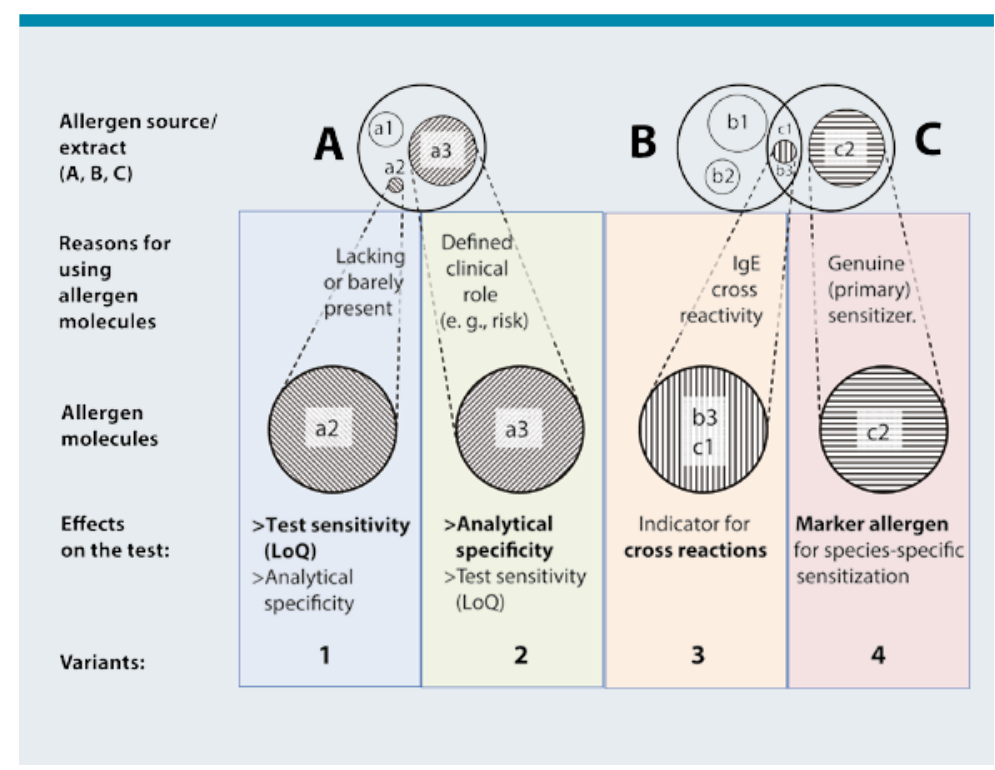

Fig. 4: Methodological rationales for molecular allergy diagnostics. The variants $1-4$ shown in the figure (see also Tab. 2 ) reflect universal arguments for the methodological use of allergen molecules. They move (virtually) exclusively on the test (sensitization) level, irrespective of the patient's clinical status. Thus, they improve only the sensitization test per se, without affecting clinical test findings/interpretation, which must always be undertaken by the treating physician (or person requesting the test) based on clinical information provided by the patient (history/provocation) on a case-by-case basis (adapted from [14]) 
Tab. 2: Improved test characteristics using defined allergen molecules for specific immunoglobulin-E (IgE) determination in a singleplex assay (see also Fig. 4 for variants) a (adapted from [14])

\section{Variants \\ Examples (allergen source, allergen carrier)}

Cat

Hazelnut

Cor a 1 (Bet v 1 homolog)

sensitivity due to lower limit of quantitation (LoQ)

(1)

Improved analytical specificity (selectivity)

Fel d 2

Cor a 14 (2S albumin)

Cor a 9 (11S globulin)

Cor a 8 (LTP, Mediterranean

region)

Kiwi

Peach

Peanut

Soy

Wheat

Meat

Bee venom

Wasp venom

Birch (hazel, alder, birch pollen)

and beech family (beech, oak

pollen)

Oleaceae (ash, olive pollen)

Poaceae (sweetgrass pollen)

Mugwort pollen

Ragweed pollen
Ara h 10

Ara h 11 (oleosins)

Gly $m 4$ (Bet v 1 homolog)

Tri a 19 ( $\Omega-5$ gliadin $)$

$a-G A L$

Ves v 5

Pru p 1 (Bet v 1 homolog)

Pru p 3 (LTP, marker, Mediterranean region)

Ara h 1 (7S globulin)

Ara h 2 (2S albumin)

Ara h 3 (11S globulin)

Ara h 6/7 (2S albumin)

Ara h 9 (LTP, Mediterranean

region)

Gly $\mathrm{m} 5$

Gly $\mathrm{m} 6$

$a-G A L$

Api $m 1$

Api $m 3$

Api $m 4$

Api $\mathrm{m} 10$

Ves $v 1$

Ves $\vee 5$

Bet v 1

Ole e 1

Phl p 1

Phl p 5

Art v 1

Amb a 1
Act d 8 (Bet-v-1-homolog)
3

Cross-reactive allergens

Fel d 2

Act d 8 (Bet-v-1-homolog)

Pru p 1 (Bet-v-1-homolog)

Pru p 4 (Profilin)

Ara h 8 (Bet v 1 homolog)

Ara $h 5^{\text {b }}$
Species-/familyspecific marker allergens

Fel d 1
Api $m 1$

Api $m 3$

Api $m 4$

Api $\mathrm{m} 10$

Ves $v 1$

Ves $v 5$

Bet v 1

Ole e $2^{b}$

Ole e $3^{c}$

Phl p $12^{\mathrm{b}}$

Phl p 7c

Phlp 5

Art v $4^{\mathrm{b}}$

Art $\vee 5^{c}$

Art v 1 Amb a 1

LTP, lipid transfer protein.

The benefit of allergen molecules as diagnostic reagents from different allergen sources/extracts (left column), the rationales, and potentially improved test characteristics (top line) vary and depend on the individual diagnostic question and the specific allergen used.

bProfilin family members: widespread, highly conserved, and extremely cross-reactive panallergens in pollen and plant-based foods.

cPolcalcin family members (pollen $\mathrm{Ca}^{++}$-binding proteins): widespread, highly conserved, and extremely cross-reactive panallergens in pollen. 
affected individuals with potential cross-reactions).

4. Depending on findings, particular allergen molecules are suitable as protein-family or speciesspecific IgE-binding marker allergens to detect or exclude genuine ("primary") sensitization.

It should be noted here that all the above-mentioned arguments move primarily on the level of sensitization and do not take the clinical status of the patient into consideration.

\section{Clinical evaluation: diagnostic sensitivity and specificity}

Diagnostic sensitivity and specificity relate to the symptoms of the affected allergy sufferer. A precondition to assessing and calculating these is: unequivocal clinical information from the patient or, in case of doubt, additional provocation tests to confirm the clinical diagnosis (Table 3, right column).

However, allergen-specific IgE diagnostics only cover sensitization (susceptibility to allergy) and cannot per se predict the clinical reaction $[12,14]$. Therefore, concordant results (positive history and positive specific IgE), for instance, are often referred to as clinically relevant (instead of correctly positive). The same applies to concordant negative results that exclude allergy, and thereby also an underlying sensitization. A positive IgE finding combined with a negative history is often classified as clinically irrelevant (instead of false positive). Declaring clinically irrelevant results as false positive does not go to the core of the matter, since ultimately the test result, i.e., the allergen-specific IgE that is present, can very well be valid and should not be questioned in the first place.

A number of clinical studies have investigated the diagnostic sensitivity and specificity of individual allergens from one allergen source. By increasing test sensitivity (low LoQ), absent or under-represented allergens were also able to significantly in crease diagnostic sensitivity. However, increased sensitizations were reported parallel to this, even in individuals with no clinically relevant reactions.

The interdependence between diagnostic sensitivity and specificity is a fundamental problem in testing and is often presented in receiver operating characteristic (ROC) curves. Better diagnostic sensitivity and specificity for the risk assessment of severe clinical reactions has been described for some single allergens, such as Ara h 2 or other highrisk allergens from the $2 \mathrm{~S}$-albumin group of storage proteins (overview in [20]). Moreover, predictive specific IgE decision points for positive or negative oral provocation in children with suspected peanut or hazelnut allergy have been defined with the help of risk-related $2 S$ albumins [21].
It must be borne in mind in relation to these complex clinical investigations that a clinical reaction (or absence thereof) can never be predicted in a foolproof manner (to $100 \%$ ) using sensitization tests such as IgE determination [21]. Therefore, methodological arguments first need to be considered for future assessments of the diagnostic suitability of allergen molecules (Tab. 3, left column). Even without a complete clinical evaluation (including diagnostic sensitivity and specificity, as well as predictive values; Tab. 3, right column) the analytical test characteristics of IgE diagnostics using allergen molecules is, in many cases, significantly better compared with allergen extracts [22]. This viewpoint is reflected in updated international laboratory guidelines on IgE test methods [14] and should serve to ease and accelerate the evaluation and introduction of allergen molecules for diagnostic purposes in the future.

\section{Interpretation to establish clinical relevance}

Ultimately, the central question relates to the clinical relevance of the specific IgE concentrations obtained:

_ The following basic rule still applies: a finding of positive specific IgE is consistent with a sensitization that is only clinically relevant in the presence of corresponding symptoms.

- A negative specific IgE finding (e.g., to an allergen molecule or a mixture of natural isoforms of a single allergen) largely excludes allergic sensitization to the tested allergen; however, only if: — Total IgE is sufficiently high

- The allergen is available intact and in adequate quantities

_ The analytical test sensitivity of the IgE determination method has been optimized and is sufficiently high

Finally, irrespective of whether allergen extracts or molecules are used for diagnostic purposes, only a physician can determine the clinical relevance of an allergic sensitization, not the test.

Therefore, all diagnostic findings from sensitization tests-and that applies equally to allergen molecules-need to be evaluated in the clinical context and on the basis of the individual patient's previous history.

\section{Potentials and quantitative concepts of molecular allergology}

Diagnostic methods using single allergens provide new opportunities to differentiate the IgE response to certain allergen sources. Some marker allergens are characteristic of certain allergen sources and enable their unequivocal classification. These triggers of genuine, primary sensitization are also referred to as species-specific allergens and can be used as "markers" for certain allergen sources. Thus, in this 
Tab. 3: General criteria for optimizing tests and universal arguments to support the use of allergen molecules in specific immunoglobulin $\mathrm{E}$ (IgE) determination ${ }^{\mathrm{a}, \mathrm{b}}$

\begin{tabular}{|c|c|c|c|}
\hline \multicolumn{2}{|r|}{ Analytical criteria (for possible test optimization) } & \multicolumn{2}{|c|}{ Clinical criteria (potential clinical advantages) } \\
\hline 1 & $\begin{array}{l}\text { Test sensitivity } \uparrow \\
\text { Limit of quantitation (LoQ) } \downarrow\end{array}$ & I & Diagnostic sensitivity $\uparrow$ \\
\hline 2 & Analytical specificity $\boldsymbol{\uparrow}$ & II & Diagnostic specificity $\uparrow$ \\
\hline 3 & Indicator of serological cross-reactivity & III & Indicator for clinical cross-reactivity \\
\hline 4 & Marker for primary/genuine sensitization & IV & Prediction of clinically relevant reactions (PPV, NPV) \\
\hline \multicolumn{4}{|c|}{$\begin{array}{l}\text { bTo what extent single allergens can optimize diagnostic/clinical criteria (right column, I-IV) depends on the cohort investigated, the single } \\
\text { allergens in question, and the study endpoints selected. In general, clinical criteria are based on the individual interpretation of test results on the } \\
\text { basis of clinical history and, where appropriate, reproducible symptoms in the affected allergy sufferer. Thus, they go beyond the actual results of } \\
\text { allergen-specific IgE tests (sensitization, yes or no). Diagnostic/clinical criteria (right column), therefore, are: less suited to the evaluation of } \\
\text { sensitization tests (hence the gray font), often not at all necessary to demonstrate the benefits of single allergens, and fraught with unsatisfactory } \\
\text { results due to their limited ability to predict clinical results. }\end{array}$} \\
\hline
\end{tabular}

part of the world for example, it is possible to reliably detect sensitizations to pollen using marker allergens and to exclude potential cross-reactions.

This is particularly useful in the case of additional sensitizations to panallergens from the polcalcin and profilin families, in order to re-establish the analytical specificity of exclusively extract-based diagnosis that is otherwise inadequate in this setting. Polcalcins and profilins are present in a wide variety of allergen sources and, due to their high structural similarity, are responsible for marked cross-reactions. Although rarely of clinical relevance, they complicate specific diagnosis when extracts alone are used, since the latter contain both marker and cross reactive allergens.

As part of test interpretation, primary sensitization in the case of a series of positive IgE results can be deduced from in the level of IgE concentrations:

The primary sensitizing allergen has the most epitopes recognized by specific IgE antibodies. In contrast, the number of cross-reactive epitopes of structurally related, similar protein allergens is often lower and/or the epitopes of lower affinity.

The following rule of thumb applies: The highest IgE level to a protein compared with other members of the same protein family likely reveals the primary sensitizer.

\section{The use of singleplex IgE tests in Bet v 1-related cross-reactivity}

A classic example of this is the PR-10 protein family, in the case of which primary birch pollen sensitization is evidenced by high Bet $\mathrm{v}$ 1-specific IgE levels, while Bet v 1-related secondary pollen or food sensitizations are reflected in low IgE values to the relevant Bet $\mathrm{v}$ 1-homologous PR-10 proteins (Fig. 5b). Moreover, the structural relationship between allergens in a family can be indirectly ascertained from the level of specific IgE (Fig. 5b).

\section{The use of singleplex IgE tests in profilin sensitization}

In the case of strong structural similarity and marked cross-reactivity within an allergen family, one can expect comparable specific IgE levels to the individual proteins, as observed with profilins for example (Fig. 5c). Determining IgE to profilins from different allergen sources is unlikely to bring any benefit here. A single IgE measurement, e.g., to grass pollen profilin $\mathrm{Phl} p 12$ or birch pollen profilin Bet $\mathrm{v} 2$, is sufficient. It is possible to establish the clinical relevance of IgE sensitization by means of detailed patient interviews: e.g., potential symptoms induced by botanically unrelated pollen plants or reactions to plant-based foods that, in particular, do not belong to the Bet-v-1 cluster, e.g., melon, banana, exotic and citrus fruits [23].

\section{The use of singleplex IgE tests against storage proteins}

IgE levels against members of the same protein family can vary significantly in the case of low structural similarity and correspondingly low cross-reactiv- 
ity, as can be seen with the example of storage proteins (Fig. 5a).

Although the typical basic structure of storage proteins, i.e.,:

$-2 S$ albumins

-7S globulins

_ 11S globulins

from different allergen sources-such as legumes (peanut, soybean), tree nuts (hazel and walnut), and seeds-is similar, only partially cross-reactive, potential IgE-binding epitopes are present. As a result, a complex pattern of possible cross-reactivities emerges, depending on the individual IgE repertoire. The IgE response to one storage protein (e.g., Ara $\mathrm{h} 3$ from peanut) does not permit an assessment of $\mathrm{IgE}$ reactivity to other members of the $11 \mathrm{~S}$ globulin family (e.g., Gly m 6 from soybean or Cor a 9 from hazelnut). Thus, strictly speaking, the sensitization pattern to storage proteins can only be determined by using all available proteins from this storage protein family. Unfortunately, not all members of these stable allergens from tree nuts, capsule and stone fruits, as well as seeds are as yet available, meaning that gaps remain in our diagnostic potential for the time being.

As a result, the highest IgE level to a particular storage protein (e.g., Ara h 2 from the $2 \mathrm{~S}$ albumin group) likely reveals the primary source of sensitization (e.g., peanut). Lower levels, e.g., to corresponding soy (2S albumin Gly $\mathrm{m}$ ) or hazel nut allergens (2S albumin Cor a 14) signal potential IgE cross-reactivity. However, their clinical relevance and the associated risk of reactions following consumption of the respective allergen source cannot be established from the level of specific IgE, but needs instead to be conclusively established by the patient's history or challenge tests.

Higher than expected IgE levels (to a food protein investigated as a secondary allergen source) raise doubts about the suspected primary allergen source and should be carefully investigated for plausibility.

Only when the corresponding proteins from the same protein family yield wholly negative IgE values can one assume that serological cross-reactivity is absent and that no clinical (cross-) reactions are to be expected.

Thus, a negative result is particularly important for the exclusion of an allergic (cross-) reaction.

It is here that the current limitations of molecular allergy diagnostics become apparent, since a structural relationship between allergens, depending on individual IgE repertoires, can determine highly variable cross-reactivities: from completely absent to strong IgE binding of similar epitopes. The various serological and clinical reaction patterns are ultimately based on numerous variables that go beyond the purely structural characteristics of the allergens:
_ Personal IgE repertoires with individual patterns of serological and potential clinical cross-reactions

_ Proportion of the allergen relative to the total protein or total weight

_ Stability of the relevant allergens, which depends on the processing of the food

- Volume of the food consumed

-Co-factors for a systemic or anaphylactic reac-

tion.

Against the background of these factors, efforts to make successful clinical predictions on the basis of molecule-specific IgE sensitizations are limited in their scope. It is essential, therefore, to correct disproportionate expectations of molecular diagnostics. IgE sensitization tests can be optimized using defined allergens and plausible criteria (predominantly independently of the clinical phenotype). The advantages for serological diagnosis,

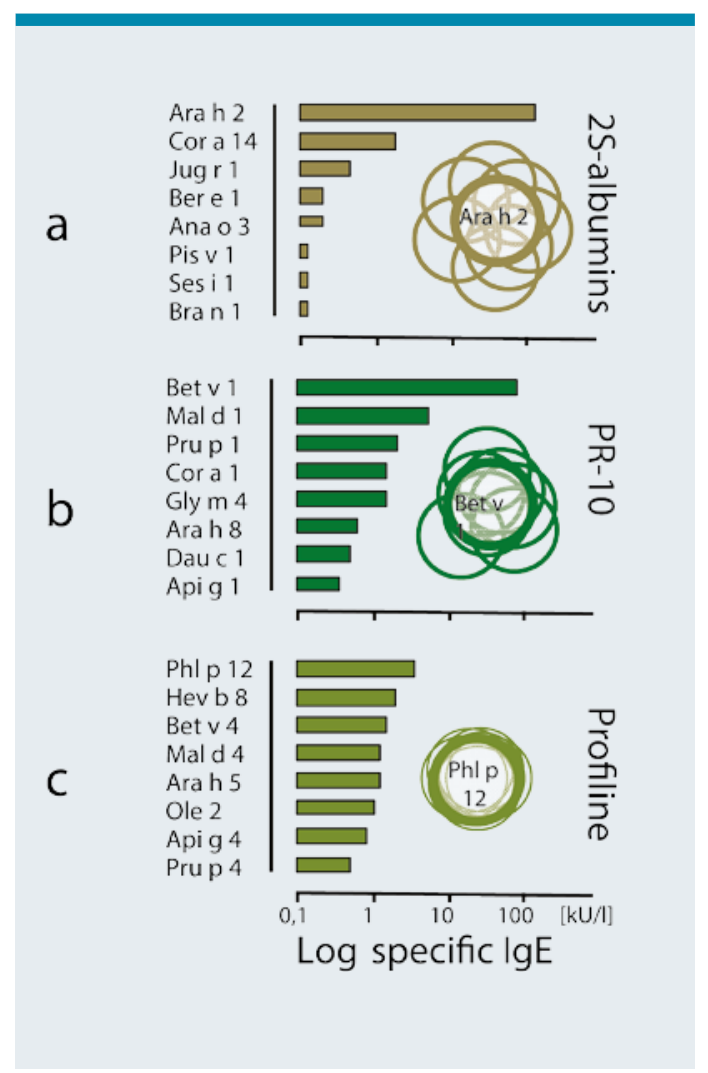

Fig. 5: Immunoglobulin E (IgE) levels to allergen molecules depending on structural similarity within an allergen family. a Variable, limited cross-reactivity between $2 \mathrm{~S}$ albumins (stable storage proteins in nuts, pulses, and seeds). b Variable cross-reactivity between Bet $v$ 1-homologous food allergens. c High crossreactivity due to the strongly preserved and similar structure of profilins (in pollen, latex, and foods) 
however, have to be demonstrated for each allergen separately.

\section{Conclusions for clinical practice}

Singleplex determinations of allergen-specific $\operatorname{IgE}$ against allergen molecules enable sensitization (i.e., allergic disposition) to be detected or excluded in a targeted manner. The novel opportunities offered by molecular allergology_increased test sensitivity and superior analytical specificity, marker function for primary sensitizations, and indicator function for serological cross-reactions-improve test characteristics, thereby broadening the opportunities offered hitherto exclusively extract-based diagnostics. Thus, carefully defined allergen molecules serve as a useful complement to the reagents available to date, and optimize IgE determinations and the detection of specific sensitization in the context of allergy diagnosis.

Our additional knowledge on molecular relationships will enable a more comprehensive and specific interpretation of variable IgE repertoires and sensitization patterns on the basis of singleplex determinations and make counseling easier. A prerequisite of this, however, is that the clinical relevance of these findings continues to be consistently determined based on individual symptoms and clinical reactions in the affected patient on a case-by-case basis.

\section{PD Dr. Jörg Kleine-Tebbe}

Allergy \& Asthma Center Westend

Outpatient Clinic Hanf, Ackermann \& Kleine-Tebbe

Spandauer Damm 130, Haus 9

14050 Berlin, Germany

email: kleine-tebbe@allergie-experten.de

Prof. Dr. Thilo Jakob

E-Mail: thilo.jakob@uniklinik-freiburg.de

\section{Conflict of interests}

Jörg Kleine-Tebbe has received research support and honoraria for further training lectures from $\mathrm{Dr}$. Fooke Gmbh and Phadia/ThermoFisher Gmbh. Thilo Jakob has received research support from Phadia/ThermoFisher $\mathrm{GmbH}$, Freiburg, and Dr. Fooke $\mathrm{GmbH}$, Neus, as well as honoraria for lectures and consultancy services from Phadia/ThermoFisher GmbH, Freiburg.

Cite this as

Kleine-Tebbe J, Jakob T. Molecular allergy diagnostics using IgE singleplex determinations: methodological and practical consideration for use in clinical routine - Part 18 of the Series Molecular Allergology. Allergo J Int 2015;24: 185-97

DOI: $10.1007 /$ s40629-015-0067-z

\section{References}

1. Schmitz R, Ellert U, Kalcklosch M, Dahm S, Thamm M. Patterns of sensitization to inhalant and food allergens findings from the German Health Interview and Examination Survey for Children and Adolescents. Int Arch Allergy Immunol 2013;162:263-70

2. Haftenberger $M$, Laussmann D, Ellert U, Kalcklosch $M$, Langen $U$, Schlaud $M$ et al. Prevalence of sensitisation to aeraoallergens and food allergens: results of the German Health Interview and Examination Survey for Adults (DEGS1). Bundesgesundheitsbl Gesundheitsforsch Gesundheitsschutz 2013;56:687-97

3. Johansson SG. The History of IgE: From discovery to 2010. Curr Allergy Asthma Rep 2011;11:173-7

4. Christensen LH, Holm J, Lund G, Riise E, Lund K. Several distinct properties of the IgE repertoire determine effector cell degranulation in response to allergen challenge. J Allergy Clin Immunol 2008;122:298-304

5. MacGlashan D Jr, Xia HZ, Schwartz LB, Gong J. IgE-regulated loss, not lgE-regulated synthesis, controls expression of FcepsilonRI in human basophils. J Leukoc Biol 2001;70:207-18

6. MacGlashan D Jr. IgE and FcepsilonRI regulation. Clin Rev Allergy Immunol 2005;29:49-60

7. Kleine-Tebbe J, Erdmann S, Knol EF, MacGlashan DW Jr, Poulsen LK, Gibbs BF. Diagnostic tests based on human basophils: potentials, pitfalls and perspectives. Int Arch Allergy Immunol 2006;141:79-90

8. Purohit A, Laffer S, Metz-Favre C, Verot A, Kricek F, Valenta R, Pauli G. Poor association between allergen-specific serum immunoglobulin E levels, skin sensitivity and basophil degranulation: a study with recombinant birch pollen allergen Bet $\mathrm{v} 1$ and an immunoglobulin $\mathrm{E}$ detection system measuring immunoglobulin $\mathrm{E}$ capable of binding to Fc epsilon RI. Clin Exp Allergy 2005;35:186-92

9. Lund G, Willumsen N, Holm J, Christensen LH, Wurtzen $\mathrm{PA}$, Lund K. Antibody repertoire complexity and effector cell biology determined by assays for IgE-mediated basophil and T-cell activation. J Immunol Methods 2012;383:4-20

10. Kleine-Tebbe J. Old questions and novel clues: complexity of IgE repertoires. Clin Exp Allergy 2012;42:1142-5

11. Ruëff F, Bergmann KC, Brockow K, Fuchs T, Grübl A, Jung Ket al. Hauttests zur Diagnostik von allergischen Soforttyp-Reaktionen. Allergo J 2010;19:404-15

12. Renz H, Biedermann T, Bufe A, Eberlein B, Jappe U, Ollert M et al. In-vitro-Allergiediagnostik. Allergo J 2010;19:11028

13. Uyttebroek AP, Sabato V, Faber MA, Cop N, Bridts CH, Lapeere $\mathrm{H}$ et al. Basophil activation tests: time for a reconsideration. Expert Rev Clin Immunol 2014;10:1325-35

14. Hamilton RG, Matsson PNJ, Chan S, Cleve M van, Hovanec-Burns D, Magnusson C et al. Analytical performance characteristics, quality assurance and clinical utility of immunological assays for human immunoglobulin $\mathrm{E}(\mathrm{lgE})$ antibodies of defined allergen specificities. 3rd ed., I/ LA20-A3, International CLSI-Guideline; 2015; in press

15. Kober A, Perborn H. Quantitation of mouse-human chimeric allergen-specific IgE antibodies with ImmunoCAP technology. J Allergy Clin Immunol 2006;117:S219 (Abstract 845 ) 
16. Hamilton RG, MacGlashan DW Jr, Saini SS. IgE antibody-specific activity in human allergic disease. Immunol Res 2010;47:273-84

17. Valenta R, Lidholm J, Niederberger V, Hayek B, Kraft D, Gronlund $\mathrm{H}$. The recombinant allergen-based concept of component-resolved diagnostics and immunotherapy (CRD and CRIT). Clin Exp Allergy 1999;29:896-904

18. Kleine-Tebbe J, Jappe U. Molekulare Allergologie - Einführung mit kommentierten Kasuistiken. München-Deisenhofen: Dustri; 2014

19. Armbruster DA, Pry T. Limit of blank, limit of detection and limit of quantitation. Clin Biochem Rev 2008;29 (Suppl 1):S49-52
20. Lange L, Beyer K, Kleine-Tebbe J. Molekulare Diagnostik bei Erdnussallergie. Allergo J Int 2014;23:158-63

21. Beyer K, Grabenhenrich L, Hartl M, Beder A, Kalb B, Ziegert $M$ et al. Predictive values of component-specific lgE for the outcome of peanut and hazelnut food challenges in children. Allergy 2015;70:90-8

22. Kleine-Tebbe J, Meißner AM, Jappe U, Herold DA. Allergenfamilien und molekulare Diagnostik IgE-vermittelter Nahrungsmittelallergien: von der Theorie zur Praxis. Allergo J 2010;19:251-63

23. Santos A, Ree R van. Profilins: mimickers of allergy or relevant allergens? Int Arch Allergy Immunol 2011;155:191204 